\title{
Effect of osmolarity of artificial tear drops on relief of dry eye symptoms: BJ6 and beyond
}

\author{
P WRIGHT, M COOPER, AND A M GILVARRY \\ From the External Diseases Clinic, Moorfields Eye Hospital, London
}

SUMMARY Patients with clinically well defined keratoconjunctivitis sicca (KCS) participated in two trials of hypo-osmolar tear substitutes. The trials were double masked, single crossover studies with computer generated random order allocation and were conducted by postal questionnaire. In the first trial a hypo-osmolar formulation using polyvinylpyrrolidone and hydroxyethyl cellulose was compared with the patients' usual treatment (BJ6 or hypromellose) and found to be inferior, especially in respect of blurring and stickiness caused by higher viscosity. In the second trial hypoosmolar preparations of BJ6 and hypromellose were compared with their iso-osmolar equivalents and found not to be significantly different in their effects. Hypo-osmolarity alone does not seem to guarantee relief of symptoms in KCS. Other factors including viscosity and colloid osmotic pressure may be more significant.

Keratoconjunctivitis sicca is a well recognised cause of much ocular pain and irritation, and, although many different solutions have been suggested as tear replacements, no single formulation has been shown to be superior for relief of symptoms. Attempts have been made to modify the composition of artificial tear drops to give maximal relief of dry eye symptoms, and some broad principles have emerged, especially relating to $\mathrm{pH} .{ }^{1-3}$ Recent studies have suggested that increased tear osmolarity is responsible for the symptoms, signs, and possibly the epithelial changes seen in keratoconjunctivitis sicca. ${ }^{48}$ Tear diluents have been suggested as a form of treatment and a small study carried out to show possible benefit. ${ }^{9}$ The present study was designed to assess whether drops with lower osmolarity but identical $\mathrm{pH}$ would give greater symptomatic relief in KCS.

\section{Material and methods}

TRIAL DESIGN

Two trials of hypo-osmolar tear substitutes were carried out. Patients with proved KCS who had been followed up for a minimum of six months in the External Diseases Clinic were selected. Diagnosis was based on history, symptoms, and signs including

Correspondence to Peter Wright, FRCS, Moorfields Eye Hospital, City Road, London EC1V 2PD. tear film abnormalities, rose Bengal staining, and grossly deficient Schirmer test $(<5 \mathrm{~mm})$ on multiple visits. Exclusion criteria were stringent, and all patients using preservative-free drops or requiring them less often than four times daily as well as those with cicatrising conjunctival disease, epithelial metaplasia, or specific types of postinflammatory corneal pathology were excluded. The trials were double masked, neither doctor nor patient being aware of which preparation was in use or in which order, with a single crossover after two weeks' treatment with each drop. The drops were posted to the patients with a questionnaire designed to allow evaluation of immediate or later effects on a wide range of symptoms commonly experienced in KCS as well as any untoward effects.

The two treatment groups were equivalent in sex, age, and treatment prior to the trials. The ratio male/female was $1 / 4$ and the mean age 51 years. The patients had previously been using hypromellose or BJ6 in a ratio of 3/1.

\section{MATERIALS}

For the first trial a new hypo-osmolar drop was formulated using two polymers different from those in our usual artificial tears but similar to many commercial preparations and previously found to be acceptable thickening agents ${ }^{10}$ (Table 1$)$.

When the results of the first trial were known it was 
Table 1 Hypodrops $M k 1$

\begin{tabular}{lc}
\hline Polyvinylpyrrolidone & $0.5 \%$ \\
Hydroxyethylcellulose & $0.5 \%$ \\
Sodium bicarbonate & $0.4 \%$ \\
Potassium chloride & $0.025 \%$ \\
Chlorhexidine acetate & $0.01 \%$ \\
Distilled water to & $100 \%$ \\
pH $\approx 8 \cdot 4$ & \\
Osmolarity $\simeq 135$ mosm/1 by freezing point depression & \\
Colloid osmolarity approximately equal one to another & \\
\hline
\end{tabular}

decided to create hypo-osmolar formulations of the usual artificial tear substitutes BJ6 and hypromellose to allow closer comparison (Table 2 and 3 ).

\section{Results}

Twenty-nine patients took part in the first trial using Hypodrops Mk 1 versus their usual drops of either BJ6 or hypromellose. Twenty-six questionnaires were completed and returned. Of these, two were spoiled and unusable, two gave no preference, and of the remainder $17(77 \%)$ preferred their old drops and only five the new ones. There was no difference between the groups in the sex ratio, order of drops used, type of symptoms usually experienced, or specific effects on symptoms, but 6/17 complained of blurring and 5/17 of stickiness while using the new drops.

These complaints appeared to be related to an increased viscosity of the new drops compared with the old, and preference for the old drops was significant at $1-5 \%$ by Fisher's test.

Table 2 Hypodrops Mk 2 (hypromellose)

\begin{tabular}{ll}
\hline Hypromellose & Hypodrop HM \\
\hline $0 \cdot 45 \%$ Sodium chloride & - \\
$0 \cdot 37 \%$ Potassium chloride & $0 \cdot 37 \%$ \\
$0 \cdot 19 \%$ Sodium borate & $0 \cdot 19 \%$ \\
$0 \cdot 19 \%$ Boric acid & $0 \cdot 19 \%$ \\
$0 \cdot 3 \%$ Hydroxypropylmethylcellulose & $0 \cdot 3 \%$ \\
$0 \cdot 1 \%$ Benzalkonium chloride & $0 \cdot 01 \%$ \\
Distilled water to $100 \%$ & \\
pH $\approx 8 \cdot 4$ & \\
Osmolarity $\simeq 158$ mosm/1 by freezing point depression \\
Colloid osmolarity approximately equal one to another \\
\hline
\end{tabular}

Table 3 Hypodrops Mk 2(BJ6)

\begin{tabular}{lll}
\hline BJ6 & Hypodrop BJ6 \\
\hline $0.6 \%$ & Sodium chloride & $0 \cdot 15 \%$ \\
$0.45 \%$ & Sodium bicarbonate & $0.45 \%$ \\
$0.25 \%$ & Hydroxypropylmethylcellulose & $0 \cdot 25 \%$ \\
$0.01 \%$ & Chlorhexidine acetate & $0 \cdot 01 \%$ \\
Distilled water to $100 \%$ & \\
pH $\approx 8 \cdot 4$ & \\
Osmolarity $\approx 160$ mosm/1 by freezing point depression \\
Colloid osmolarity approximately equal one to another \\
\hline
\end{tabular}

The second trial involved 26 patients of whom 24 returned usable completed questionnaires.

The overall preferences expressed were: isoosmolar 14, hypo-osmolar 6 , undecided 4 . The apparent preference for iso-osmolar drops was not significant at the $5 \%$ level.

Despite the random allocation of the drops there was an apparent first use effect on expressed preference. Of those who preferred the hypo-osmolar drops all six had used the hypo-osmolar preparation first. Of those preferring the iso-osmolar drops nine $(60 \%)$ had used the iso-osmolar first and only five (33\%) the hypo-osmolar. Again these findings were not statistically significant.

Replies to specific questions concerning relief of common dry eye symptoms and possible adverse effects of the drops showed no statistically significant difference for the two preparations (Table 4).

\section{Discussion}

KCS is essentially a symptomatic problem complicated in a few patients by infection, for which there is still no completely satisfactory treatment. Replacement of the missing fluid from the outer eye would seem a simple matter, but despite clinical and laboratory studies ${ }^{11}$ no universally acceptable tear substitute exists. Even though alkaline eye drops give the best subjective relief for KCS sufferers, only hypromellose and BJ6 are alkaline. Among other commercial preparations values as low as $\mathrm{pH} \mathrm{4.6}$ are found, and none has a value higher than $\mathrm{pH} 7 \cdot 50 .{ }^{12}$ Unfortunately the symptom complex caused by tear deficiency is not specific, and many other outer eye disorders, especially blepharitis and primary ocular surface disorders, may produce identical symptoms. It follows that the large number of prescriptions for artificial tear drops therefore only partly represent replacement therapy for dry eyes and probably the greater use is as a placebo. This more than any other factor explains the conflicting reports of their efficacy, and it was considered essential in this study to try the drops only in those patients with well

Table 4 Effect on symptoms

\begin{tabular}{llr}
\hline $\begin{array}{l}\text { Iso-osmolar } \\
\text { preferred }\end{array}$ & Symptoms & $\begin{array}{l}\text { Hypo-osmolar } \\
\text { preferred }\end{array}$ \\
\hline $63 \%(9)$ & Stickiness & $67 \%(4)$ \\
$36 \%(5)$ & Stinging & $67 \%(4)$ \\
$29 \%(4)$ & Burning & $50 \%(3)$ \\
$93 \%(13)$ & Grittiness & $100 \%(6)$ \\
$63 \%(9)$ & Blurring & $50 \%(3)$ \\
$43 \%(3)$ & Itching & $67 \%(4)$ \\
$50 \%(7)$ & Pricking & $83 \%(5)$ \\
$29 \%(4)$ & Pain with TV & $33 \%(2)$ \\
$50 \%(7)$ & Smoke related pain & $67 \%(4)$ \\
\hline
\end{tabular}

Differences not statistically significant 
established KCS who were dependent on tear substitute drops.

Increased osmolarity of the tears in the marginal tear strip has been found in patients with $\mathrm{KCS}^{45}$ and proposed as a useful diagnostic procedure. ${ }^{13}$ The test involves microsampling of the marginal tear meniscus and estimation of osmolarity by freezing point depression. The tear osmolarity from normal eyes averaged 302 (SD 6.3) mosm $/ 1$, and tears from KCS eyes averaged 343 (SD 32.3) $\mathrm{mosm} / 1$. With a cut-off at $311 \mathrm{mosm} / 1$, the samples differentiated with a specificity of $93.7 \%$ and a sensitivity of $94.7 \%$. There was a large variability between right and left eyes, and the reproducibility was not good, so considerable doubt must remain about the validity of microsampling of marginal strip tear fluid in eyes with reduced flow, altered mixing, and enhanced evaporation.

Individual and diurnal variations in tear osmolarity have been found in normal, healthy, asymptomatic adults with individual subject means ranging from 310 to $334 \mathrm{mosm} / \mathrm{kg}$. ${ }^{14}$ The finding of increased tear osmolarity in patients with dysthyroid eye disease ${ }^{15}$ also casts doubt on the prime role of hyperosmolarity in the causation of the epithelial changes seen in KCS. Although damage to rabbit corneal epithelium in vivo and in vitro has been demonstrated after exposure to solutions of hyperosmolar concentrations in the same range as is found in KCS, these rabbits do not show the clinical signs of KCS, and evidence of epithelial damage was found only at ultrastructural level. Cells showed loss of microvilli and desmosomal attachments, tending to round up or desquamate.

Hyperosmolarity of the tear fluid has been observed in rabbits after occlusion of the ducts of the tear producing glands, and the model has been suggested as a good one for the study of KCS. ${ }^{16}$ These animals do not show any of the clinical features of human KCS, and the relevance of this experimental animal model to human disease has still to be confirmed. There is also a need to collate the findings in the proposed rabbit model with the previously reported finding of different osmolarity in rabbit tear fluid from the conjunctival sac and that obtained by cannulation of the lacrimal excretory duct. It was postulated that tears in the conjunctival sac remain isotonic, with plasma-like concentrations of sodium and chloride at all flow rates because of transport of these ions across the conjunctiva. ${ }^{17}$ Why this does not occur in the rabbit dry eye model and in human subject remains unexplained.

Tear diluents have been proposed for treatment of KCS, and it has been shown that solutions of low osmolarity $(75 \mathrm{mosm} / 1)$ are irritant and the ability of any hypo-osmolar solution to reduce tear fluid osmolarity is very short lasting. ${ }^{9}$ The reported benefit of $150 \mathrm{mosm} / 1$ drops with a 2:1 preference for hypoosmolar drops has not been confirmed in our present study. It may be relevant that, among the small treatment groups, three out of four were not using artificial tear drops regularly, and so despite the osmotic evidence of KCS they could not be said to be typical dry eyed patients. The studies we now report suggest that differences of osmolarity do not override the effects of viscosity or $\mathrm{pH}$ and that when these latter are controlled no clear preference for hypoosmolar drops can be shown.

A recent publication has drawn attention to the possible effect of total colloidal osmolarity, or oncotic pressure, on the efficacy of artificial tear drops. ${ }^{18}$ Although the colloidal osmolarity of normal tears and most tear substitutes is low, one commercial preparation said to be useful because it is hypoosmolar was found to have an oncotic pressure 60 times greater than that of most other artificial tear drops. The high colloidal osmolarity of the preparation is attributed to the high content of non-ionic crystalloids, especially glucose. The drops used in the trial now reported contained matching amounts of macromolecules to give comparable colloid osmolarity.

Further studies are planned to determine whether increased colloid osmolarity with or without alkaline $\mathrm{pH}$ will offer greater symptomatic relief. Meanwhile, although there will always be considerable individual variations of preference for one of the many tear substitutes available, there do not at present seem to be any good clinical data to suggest a need to modify the formulation of $\mathrm{BJ} 6$ or hypromellose to relieve dry eye symptoms.

We are grateful to Mr V Andrews and the staff of the Pharmacy for much help in formulating the drops and to Professor F J Holly for measurements of colloid osmolarity.

\section{References}

1 Jones BR, Coop HV. The management of keratoconjunctivitis sicca. Trans Ophthalmol Soc UK 1965; 85: 379-89.

2 Wright P. Diagnosis and treatment of dry eyes. Trans Ophthalmol Soc UK 1971; 91: 119-28.

3 Raber I, Breslin CW. Toleration of artificial tears. The effect of pH. Can J Ophthalmol 1978; 13: 247-9.

4 Mishima S, Kubota Z, Farris RL. The tear flow dynamics in normal and keratoconjunctivitis sicca cases. Concilium Ophthalmologicum XXI. Mexico, 1970. Acta 2: 1801-5.

5 Gilbard JP, Farris RL, Santamaria J. Osmolarity of tear microvolumes in keratoconjunctivitis sicca. Arch Ophthalmol 1978; 96: 677-81.

6 Gilbard J, Farris RL. Tear osmolarity and ocular surface disease in keratoconjunctivitis sicca. Arch Ophthalmol 1979; 97: 1642-6.

7 Farris LR, Stuchell RN, Mandell ID. Basal and reflex tear analysis I. Physical measurements: osmolarity, basal volumes and reflex flow rate. Ophthalmology (Rochester) 1981; 88: 852-7.

8 Gilbard JP, Carter JB, Sang DN, Refojo MF, Hanninen L, Kenyon KR. Morphologic effect of hyperosmolarity on rabbit 
corneal epithelium. Ophthalmology (Rochester) 1984; 91: 1205-12.

9 Gilbard JP, Kenyon KR. Tear diluents in the treatment of keratoconjunctivitis sicca. Ophthalmology (Rochester) 1985; 92: 646-50.

10 Dudinski O, Finnin BC, Reed BL. Acceptability of thickened eye drops to human subjects. Curr Ther Res 1983; 33: 322-37.

11 Holly FJ. Surface chemistry of tear film component analogues. J Coll Interface Sci 1974; 49: 221-31.

12 Motolko M, Breslin CW. The effect of $\mathrm{pH}$ and osmolarity on the ability to tolerate artificial tears. Am J Ophthalmol 1981; 91: 781-4.

13 Farris RL, Gilbard JP, Stuchell RN, Mandel ID. Diagnostic tests in keratoconjunctivitis sicca. J Contact Lens Assoc Ophthalmol 1983; 9: 23-8.
14 Benjamin WJ, Hill RM. Human tears: osmotic characteristics. Invest Ophthalmol Vis Sci 1983; 24: 1624-6.

15 Gilbard JP, Farris RL. Ocular surface drying and tear film osmolarity in thyroid eye disease. Acta Ophthalmol (Kbh) 1983; 61: 108-16.

16 Gilbard JP, Huang A, Tseng S, Rossi S, Gray K, Hanninen L. Rabbit model for keratoconjunctivitis sicca. Invest Ophthalmol Vis Sci 1985; 26: ARVO abstracts, 14.

17 Gilbard JP, Dartt DA. Changes in rabbit lacrimal gland fluid osmolarity with flow rate. Invest Ophthalmol Vis Sci 1982; 23: 804-6.

18 Holly FJ, Esqivel ED. Colloid osmotic pressure of artificial tears. J Ocul Pharmacol 1985; 1: 327-36. 